\title{
INVESTIGACIÓN/RESEARCH
}

Recibido: 24/09/2013-----Aceptado: 21/10/2013-----Publicado: 15/03/2014

\section{COMPLEJIDAD Y SISTEMATIZACIÓN DE LA EVALUACIÓN DE LA EDUCACIÓN SUPERIOR EN MÉXICO}

Braulio González Vidaña': Universidad del Claustro de Sor Juana. Consultoría Internacional Social y Educativa (CISYE). México bgonzalez@ucsj.edu.mx

\section{RESUMEN}

Condiciones tales como la crisis de desarrollo de los años ochenta en América Latina; la masificación de la educación superior; la creciente participación y exigencia de transparencia de la sociedad civil hacia instituciones y organismos vinculados a servicios públicos como la educación; el avance de los regímenes democráticos y la difusión de la cultura de la calidad, impactaron en las instituciones de educación terciaria produciendo la necesidad de establecer modelos de gestión educativa sustentados en modelos de evaluación capaces de garantizar la consistencia de los programas educativos ofrecidos por las instituciones de educación superior con las demandas del entorno productivo, social y cultural. México no fue la excepción y desde la década de los ochentas del siglo $X X$ se impulsaron iniciativas gubernamentales, así como de organismos de la sociedad civil, que buscaron establecer modelos de evaluación y en algunos casos de acreditación de las instituciones $y / 0$ programas de educación superior. El problema es que se ha producido una gran cantidad de instituciones y exigencias de evaluación que por un lado responden a la complejidad social, cultural y educativa del país, pero por otro lado generan la urgencia de sistematizar los modelos aplicados a la fecha para no perder la posibilidad de educar con pertinencia y calidad por ocuparse sólo de evaluar. El enfoque propuesto en este artículo es evaluar para educar y no educar para evaluar.

PALABRAS CLAVE: Educación superior - Complejidad - Sistematización - Evaluación - Acreditación.

\footnotetext{
${ }^{1}$ Braulio González Vidaña: Universidad del Claustro de Sor Juana. Consultoría Internacional Social y
} 


\section{COMPLEXITY AND SYSTEMATIZATION OF EVALUATION OF EDUCATION HIGHER IN MEXICO}

\section{ABSTRACT}

Conditions such as the development crisis of the 1980s in Latin America; the massification of higher education; increased participation and call for transparency of civil society institutions and organisms linked to public services such as education; the advance of democratic regimes and the diffusion of the culture of quality, impact on tertiary education institutions producing the need to establish educational management models based on assessment models capable of ensuring the consistency of the educational programs offered by institutions of higher education with the demands of the productive, social and cultural environment. Mexico was no exception and since the Decade of the eighties of the century XX governmental initiatives, are boosted as well as civil society organizations that sought to establish evaluation models and in some cases accreditation of institutions or programmes of higher education. The problem is that there has been a large number of institutions and requirements of assessment on the one hand to meet the social, cultural and educational complex of the country, but on the other hand generate urgency to systematize models applied to date to avoid losing the possibility of teaching with relevance and quality by only dealing with assessing. The approach proposed in this article is to evaluate to educate and not educate to evaluate.

KEYWORDS: higher education; complexity; systematization; evaluation; accreditation and quality.

\section{INTRODUCCIÓN}

El propósito de este trabajo es contribuir a la reflexión que se está suscitando en México con respecto a la necesidad de establecer las bases de una propuesta de integración del Sistema Nacional de Evaluación y Acreditación de la Educación Superior que se integre a partir del reconocimiento de las propuestas diseñadas por la Asociación Nacional de Universidades e Instituciones de Educación Superior (ANUIES) y las experiencias desarrolladas por otras naciones latinoamericanas, en concreto se toma el caso de la Comisión Nacional de Evaluación y Acreditación Universitaria (CONEAU) de Argentina que es un organismo de carácter nacional con facultades de evaluación de la totalidad del sistema de educación superior en sus distintas modalidades y niveles de enseñanza.

\section{OBJETIVOS}

El problema central es que en México existen varios modelos y sistemas de evaluación y acreditación de la educación superior que no dialogan entre ellos, por lo que producen la duplicación de esfuerzos por parte de las IES (públicas y privadas) a fin de mantener las exigencias de calidad en los más altos niveles de excelencia exigibles 
a la educación superior mexicana en el marco de la sociedad global del conocimiento.

La propuesta central es analizar la viabilidad de establecer un sistema de equivalencias y/o convalidaciones de procesos de evaluación y acreditación de las IES, así como de sus programas académicos.

\section{METODOLOGÍA}

El enfoque de la presente investigación es cualitativa y se fundamenta en la revisión de los documentos normativos y metodológicos de los modelos de evaluación, acreditación y certificación de la educación superior vigentes en México. Este trabajo no pretende simplificar la complejidad real de la evaluación de la educación superior en México, sin comprender el entorno educativo a nivel internacional, así como su impacto en la práctica institucional y en los estándares de calidad educativa.

\section{RESULTADOS}

\subsection{La experiencia mexicana}

Los procesos, modelos y enfoques de evaluación de la educación superior en México se resumen en dos grandes líneas: evaluación institucional y evaluación de programas académicos. Desde ese punto de partida, surgen en México diversas propuestas de evaluación tendientes a reconocer los alcances y limitaciones de las instituciones y programas de educación superior, con el objetivo de garantizar su relevancia y pertinencia con respecto a las necesidades de desarrollo nacional, regional y local. (Rodríguez Andujo, et. Al. 2009).

No se trata de examinar aquí cada una de las leyes que se han cruzado en el camino de la educación ni de reflexionar sobre el contenido de cada una de ellas, que han sido varias, sino de criticar precisamente eso, los cambios que se han ido produciendo en nuestro modelo educativo (Fernández, 2009: 16).

A partir de la publicación de la Ley para la Coordinación de la Educación Superior en diciembre de 1978, se organizó el Sistema Nacional para la Planeación Permanente de la Educación Superior (SINAPPES), del que se desprendió una instancia de gestión denominada Coordinación Nacional para la Planeación de la Educación Superior (CONPES), así como las Comisiones Estatales para la Planeación de la Educación Superior (COEPES) surgidas en las entidades de la República que junto con las Coordinaciones Regionales para la Planeación de la Educación Superior (CORPES) generaron un conjunto de criterios de evaluación de la pertinencia de los planes y programas de estudio propuestos por instituciones de educación superior públicas y privadas de conformidad con criterios nacionales, regionales y estatales de planeación de la educación superior mexicana (Gago Huguet, et. Al. 2012).

Posteriormente, hacia el año 1984, la Asociación Nacional de Universidades e Instituciones de Educación Superior (ANUIES) aprobó un documento titulado "La evaluación de la educación superior en México" el cual, según lo informa el documento de ANUIES (Gago, et. Al. 2012): "aborda extensamente cuestiones metodológicas 
como una taxonomía de análisis y un listado de indicadores para evaluar tanto al sistema como a las IES." (Gago, et. Al. 2012: 17)

En ese mismo año se organiza el Sistema Nacional de Investigadores (SNI) que define los criterios de evaluación de la investigación producida en el país y propone un programa de estímulos a los investigadores nacionales con mejor desempeño en tres grandes niveles de reconocimiento y la categoría de "Candidato" que estimula la formación de recursos humanos de alto nivel para la investigación mexicana.

Años después, en 1989, se instala la Comisión Nacional para la Evaluación de la Educación Superior (CONAEVA) que tenía la encomienda de coordinar los trabajos de evaluación de la educación superior entre la Secretaría de Educación Pública y la ANUIES. Asimismo, se pusieron en marcha los Comités Interinstitucionales para la Evaluación de la Educación Superior (CIEES) en el año de 1991 y que desde entonces tienen la encomienda de evaluar planes y programas de estudio de nivel superior mediante una metodología sostenida en la participación de pares académicos provenientes de IES del sistema educativo nacional (Aréchiga Urtuzuástegui y Llarena de Thierry, 2003).

En 1992 se impulsa la organización del Centro Nacional para la Evaluación de la Educación Superior (CENEVAL) que tiene el objetivo de certificar a los estudiantes que ingresan y egresan de los niveles medio superior y superior de México mediante la elaboración de exámenes de ingreso (EXANI) y egreso (EGEL) a estos dos niveles educativos. (Gago, 2002)

Con el surgimiento en 1994 del Consejo de Acreditación para la Enseñanza de la Ingeniería (CACEI) inició una etapa de creación de organismos acreditadores especializados en planes y programas de estudio de IES públicas y privadas, que darían pie al surgimiento del Consejo para la Acreditación de la Educación Superior, A.C. (COPAES) en el año 2000. El COPAES asume la responsabilidad de autorizar los servicios de acreditación que ofrecen los organismos evaluadores y acreditadores de programas académicos (Gago, 2002).

En 1994 la Federación de Instituciones Mexicanas Particulares de Educación Superior, A. C. (FIMPES) puso en marcha un sistema de ingreso y permanencia para las instituciones particulares interesadas en ser parte de FIMPES y para aquellas que se encuentran afiliadas. El sistema se integra con el objetivo de acreditar a las instituciones en su totalidad, desde la aplicación de su filosofía institucional hasta su viabilidad financiera, pasando por los programas académicos y el cuerpo docente. Hasta ahora, el modelo de FIMPES es el único en México que evalúa en la perspectiva institucional y desde entonces se ha actualizado en tres ocasiones para dar lugar a un modelo de evaluación tendiente a reconocer y acreditar los resultados en el aprendizaje de los egresados de las instituciones de educación superior (FIMPES, 2013).

Por otra parte, las IES con financiamiento público trabajan en los Programas Integrales de Fortalecimiento Institucional (PIFI) que año con año deben elaborar para acceder a recursos extraordinarios que les permitan impulsar su desarrollo más 
allá de las limitaciones presupuestarias impuestas por el alto costo del gasto corriente que las instituciones públicas cargan (López Zárate, 2012).

Asimismo y para concluir con esta panorámica del estado que guarda la evaluación y acreditación de la educación superior en México, es importante señalar que existe un Programa Nacional de Posgrados de Calidad al que se someten los programas académicos de dicho nivel educativo tanto de instituciones públicas como aquellos ofrecidos por las instituciones de educación superior con financiamiento privado.

El conjunto de organismos, sistemas y modelos de evaluación, acreditación y certificación de la educación superior -sin considerar la aplicación de procesos de certificación de procesos de calidad por medio de las normas ISO- constituyen un entramado complejo, heterogéneo y multidimensional de la evaluación en México que implica altos costos financieros, administrativos y humanos para las IES mexicanas, sin quedar suficientemente claro su impacto real en la mejora de los procesos educativos y su impacto en los aprendizajes adquiridos por los egresados de la educación superior mexicana.

\subsection{La experiencia latinoamericana}

Conforme a lo analizado por de la Garza (2008) el acontecimiento que detona lo que él denomina la "década de la calidad en la educación superior en América Latina y el Caribe" fue la llamada "Declaración sobre la Educación Superior en América Latina y el Caribe" firmada en La Habana, Cuba en noviembre de 1996 y que fue firmada por 26 países de la región que proclamaron lo siguiente en la Conferencia Regional sobre la Educación Superior (CRES 96) según lo cita Javier de la Garza:

"El conocimiento es un bien social que sólo puede ser generado, transmitido, criticado y recreado, en beneficio de la sociedad, en instituciones plurales y libres, que gocen de plena autonomía y libertad académica, pero que posean una profunda conciencia de su responsabilidad y una indeclinable voluntad de servicio en la búsqueda de soluciones a las demandas, necesidades y carencias de la sociedad, a la que deben rendir cuentas como condición necesaria para el pleno ejercicio de la autonomía. La educación superior podrá cumplir tan importante misión en la medida en que se exija a sí misma la máxima calidad, para lo cual la evaluación continua y permanente es un valioso instrumento" (de la Garza, 2008: 185-186).

En 1998, la Conferencia Mundial sobre Educación Superior celebrada por la UNESCO en París emite una Declaración Mundial que sostiene con respecto a la calidad de la educación superior y que a continuación recupera de la Garza (2008):

"La calidad de la enseñanza superior es un concepto pluridimensional que debería comprender todas sus funciones y actividades: enseñanza y programas académicos, investigación y becas, personal, estudiantes, edificios, instalaciones, equipamiento y servicios a la comunidad y al mundo universitario. Una autoevaluación interna y un examen externo realizados con transparencia por expertos independientes, en lo posible especializados en lo internacional, son esenciales para garantizar la calidad. 
Deberían crearse instancias nacionales independientes, y definirse normas comparativas de calidad, reconocidas en el ámbito internacional. Con miras a tener en cuenta la diversidad y evitar la uniformidad, debería prestarse la atención requerida a las particularidades de los contextos institucional, nacional y regional. Los protagonistas den ser parte integrante del proceso de evaluación institucional" (De la Garza, 2008: 187).

En el viejo continente se destaca en repetidas ocasiones la necesidad de no limitarse a los aspectos puramente económicos del desarrollo, sino a considerar la realización personal del individuo como una de los objetivos esenciales de la educación y la formación (Peña, 2003: 53)

A continuación se revisará una de las experiencias más exitosas de sistematización nacional de la evaluación y la acreditación de la educación superior: la de la Comisión Nacional de Evaluación y Acreditación Universitaria (CONEAU) de Argentina, que de la mano con el Consejo Nacional de Acreditación (CNA) de Colombia y el Sistema Nacional de Acreditación de la Educación Superior (SINAES) de Costa Rica, integran prácticas de evaluación y acreditación institucional y de programas que bien podrían contribuir a pensar en las posibilidades de un sistema semejante en México.

Javier de la Garza (2008) explica que existen tres tipos de sistemas de acreditación en América Latina y el Caribe:

"a) los organismos gubernamentales; b) los organismos intermedios; c) los organismos profesionales/académicos. La tendencia es establecer organismos gubernamentales, con un número creciente de elementos, de estructuras y prácticas desarrolladas por los tipos intermedios, pero sobre todo los de carácter académico." (de la Garza, 2008: 191)

Cabe señalar que, no obstante la heterogeneidad de modelos de organización vigentes en la región, la mayor parte los procesos de evaluación y acreditación cumplen con tres grandes etapas (De la Garza, 2008):

1) Autoevaluación o autoestudio como fundamento de la totalidad del proceso y mecanismo para mejorar el autoconocimiento de los actores de una institución a fin de conducir acciones que fortalezcan la vida académica e institucional.

2) Evaluación externa conducida por especialistas o pares académicos que examinan la correspondencia existente entre los reportes surgidos de la etapa de autoevaluación y la realidad observada en visitas de verificación a las instalaciones de la institución sujeta a evaluación.

3) Evaluación final para emitir un dictamen de acreditación y que es realizada por expertos adscritos al organismo acreditador. Se emiten sugerencias y recomendaciones tendientes a contribuir al desarrollo de la institución y/o su programa académico. 
Retomando el caso de Argentina, según lo señalado por Fernández Lamarra (2003) la Comisión Nacional de Evaluación y Acreditación Universitaria (CONEAU) se crea en 1996 con la participación de doce miembros designados por el Poder Ejecutivo y en el marco de una Ley de Educación Superior emitida en 1995. Las funciones principales de la CONEAU son:

- Coordinar y llevar a cabo las evaluaciones externas de las instituciones universitarias, con la participación de pares académicos de reconocida competencia.

- Acreditar las carreras de grado correspondientes a profesiones reguladas por el Estado.

- Acreditar las carreras de posgrado, ya sean de especialización, maestría y doctorado.

- Expedirse sobre la consistencia y viabilidad del proyecto institucional requerido para que el Ministerio de Educación autorice la puesta en marcha de una nueva institución universitaria nacional con posterioridad a su creación por ley del Congreso Nacional o el reconocimiento de una provincial.

- Preparar los informes requeridos para otorgar o no la autorización provisoria y el reconocimiento definitivo de las instituciones universitarias privadas, así como los informes en base a los cuales se evalúa anualmente el período de funcionamiento provisorio de dichas instituciones.

- Expedirse sobre las propuestas para la constitución de entidades privadas con fines de evaluación y acreditación universitaria en forma previa a la decisión al respecto del Ministerio de Educación (Fernández Lamarra, 2003: 10).

De este modo la CONEAU nace con facultades que integran la evaluación de instituciones y de programas de grado y posgrado. Además de acreditar a estas instituciones públicas y privadas y valorar la pertinencia de abrir nuevas opciones de instituciones educativas de nivel superior. Los doce integrantes de la CONEAU los elige el Poder Ejecutivo nacional de la siguiente forma: tres son propuestos por el Consejo Interuniversitario Nacional, uno del CRUP, uno de la Academia Nacional de Educación, tres de cada una de las Cámaras del Congreso de la Nación y uno del Ministerio de Educación (Fernández Lamarra, 2003: 10).

Otro elemento interesante a considerar es que la CONEAU tiene la facultad de dictaminar a entidades privadas para conducir los procesos de evaluación y acreditación y que con el dictamen de la CONEAU se emite un reconocimiento por parte del Ministerio de Educación.

Cabe señalar que, no obstante que el proceso de autoevaluación o autoestudio es responsabilidad de cada institución, la CONEAU ofrece asesoría, capacitación y acompañamiento permanente a las instituciones que se encuentran en proceso:

"Para ello ha organizado cursos y talleres sobre aspectos conceptuales y metodológicos de estos procesos; ha celebrado acuerdos de cooperación con un alto número de instituciones; ha elaborado y difundido diversos documentos teóricometodológicos sobre aspectos específicos de la evaluación institucional; ha brindado 
asesoramiento por parte de su equipo técnico a las instituciones que lo han solicitado; etc. Es decir, una estrategia de asistencia y cooperación que tiende a fortalecer dentro del sistema universitario la denominada 'cultura de la evaluación" (Fernández Lamarra, 2003: 12).

Más allá de las debilidades analizadas por Fernández Lamarra (2003) con respecto a la CONEAU, las cuales se podrían estudiar en otro artículo de investigación que permita proponer el modelo de gestión del Sistema Nacional de Evaluación y Acreditación de la Educación Superior Mexicana, capaz de atender a la complejidad de los sistemas vigentes de evaluación y acreditación de las instituciones y/o programas de educación superior en México; el modelo de la CONEAU es una práctica exitosa que, entre otros beneficios, presenta los siguientes a la luz del que escribe estas líneas:

1. Integra las necesidades de evaluación y acreditación en los dos grandes ejes: evaluación institucional y evaluación de programas académicos en estudios de grado y posgrado.

2. El modelo CONEAU incluye la planeación y dictaminación de propuestas de apertura de nuevas instituciones de educación superior privada.

3. CONEAU tiene facultades para evaluar la educación superior en sus distintas modalidades de enseñanza, incluso educación a distancia.

4. El modelo CONEAU fortalece la cultura de la evaluación por encima de los criterios comerciales que producen los rankings o las acreditaciones como argumentos de comercialización de las IES públicas o privadas. CONEAU enfatiza la evaluación como proceso educativo y no con fines de reconocimiento de mercado.

5. CONEAU es un organismo público, con facultades reconocidas por la Ley de Educación Superior emitida en 1995 y esto permite que se convierta en una herramienta para garantizar la pertinencia social y relevancia educativa de las IES argentinas públicas y privadas.

\subsection{La propuesta de ANUIES en México}

En el documento titulado Evaluación, certificación y acreditación en la educación superior de México. Hacia la integración del Subsistema para Evaluar la Educación Superior (2012), el grupo de académicos encabezados por Antonio Gago Huguet proponen varios ejes de reforma para mejorar el funcionamiento de lo que ellos llaman Subsistema para Evaluar la Educación Superior (SEES).

El trabajo aprobado en la XXXIX Sesión Ordinaria de la ANUIES celebrada el 24 de octubre del año 2008, contiene una revisión de los esfuerzos, logros, alcances, limitaciones y retos de los siguientes organismos y programas de evaluación y acreditación: 
- Sistema Nacional de Investigadores.

- Programa Nacional de Posgrados de Calidad.

- Comités Interinstitucionales para la Evaluación de la Educación Superior.

- Consejo para la Acreditación de la Educación Superior, A.C.

- Centro Nacional de Evaluación para la Educación Superior.

- Evaluaciones que realizan las IES y las autoridades gubernamentales.

- Programa de Mejoramiento del Profesorado.

- Programa Integral de Fortalecimiento Institucional.

- Programa de Estímulos al Desempeño del Personal Docente.

Como se puede observar, el gran ausente es el sistema de acreditación institucional de la Federación de Instituciones Mexicanas Particulares de Educación Superior, A.C. (FIMPES) que, como ya se mencionó en el primer apartado de este artículo, se enfoca a evaluar a las instituciones particulares de educación superior que desean ingresar y/o permanecer como miembros afiliados a dicho organismo. Entre los factores que inciden en este desconocimiento de las acciones de evaluación y las contribuciones del sistema de FIMPES se pueden mencionar los siguientes:

- El carácter gremial del sistema de acreditación que sólo cubre a instituciones particulares, de las cuales sólo 102 son instituciones acreditadas por FIMPES.

- La divergencia de intereses de las IES particulares y las IES públicas. Sus agendas de política educativa no tienen muchos puntos de contacto, e incluso pueden confrontarse en aspectos relacionados con los fines y el financiamiento de la educación superior.

- El hecho de que no se conoce por parte de la ANUIES el fondo del modelo FIMPES para la evaluación y acreditación.

- El prejuicio existente en México con respecto a los propósitos que animan a algunas de las instituciones particulares de educación superior.

Más allá de estas condiciones, si se desea estructurar un sistema integral y multidimensional, se requiere incorporar el modelo de evaluación y acreditación de instituciones desarrollado por la FIMPES y que en México sigue siendo el único enfocado a la acreditación de instituciones (Korniejczuk, 2002). Regresando a la propuesta de la ANUIES (Gago, et. Al. 2012), ésta se concentra en los siguientes aspectos:

- Conceder mayor relevancia a la evaluación de los aprendizajes.

- Establecer una entidad de evaluación en cada IES.

- Formalizar la integración de los CIEES y el COPAES.

- Mejorar la supervisión de los organismos que acreditan programas.

- Articular marcos de referencia.

- Alinear los marcos de referencia de los organismos evaluadores con las condiciones internacionales de la educación superior. 
- Establecer regulaciones semejantes para la administración.

- Revisar las cuotas de los organismos acreditadores.

- Impulsar la formación de profesionales especializados en la evaluación educativa.

- Reforma integral del proceso dirigido por SEP federal y por las Secretarías de Educación de los estados de la República, para otorgar los Reconocimientos de Validez Oficial de Estudios (RVOE) a instituciones particulares de educación superior.

- Nueva legislación para regular el ejercicio de las profesiones.

- Propuestas para mejorar el desempeño del CENEVAL.

- Propuestas para mejorar la evaluación.

Una aclaración con respecto a la propuesta del equipo de ANUIES (Gago, et. Al. 2012) es que se habla de un Subsistema para Evaluar la Educación Superior (SEES) dependiente del Sistema Nacional para Evaluar la Educación (SNEE) comprometido por el gobierno de Felipe Calderón Hinojosa en el periodo 2006-2012. Con las recientes modificaciones a la legislación educativa, las autoridades del ramo se han enfocado a trabajar más en la educación básica y en la resolución de los conflictos sociales y políticos derivados de la Reforma Educativa de 2013 y su legislación secundaria. El nuevo papel asignado al Instituto Nacional de Evaluación Educativa (INEE) no incluye -por lo menos hasta ahora- la evaluación de la educación superior lo que hace pensar en la necesidad de reformular una política pública que fortalezca el desarrollo de la educación superior mexicana.

El núcleo de la propuesta de la ANUIES (Gago, et. Al. 2012) se puede resumir en el siguiente enunciado del equipo coordinado por Gago Huguet en relación con el fortalecimiento del papel desempeñado por la Comisión Nacional para la Evaluación de la Educación Superior (CONAEVA):

"[...] proponer que se instituya esta Comisión - de manera formal y con los sustentos jurídico y económico pertinentes- como la entidad que se ocupe de coordinar, apoyar y dar seguimiento a los programas y acciones de evaluación, certificación y acreditación que se realizan en las IES, las dependencias gubernamentales y demás organizaciones en el ámbito de la educación superior en México" (Gago, et. Al. 2012: 52).

La propuesta de la ANUIES (Gago, et. Al. 2012) resulta oportuna porque coloca en el centro de la discusión nacional sobre la educación superior, la urgencia de vertebrar el subsistema de evaluación de la educación superior desde una perspectiva integradora de las distintas experiencias, modelos y metodologías vigentes en México, con la excepción hecha del modelo FIMPES de acreditación institucional. La iniciativa contiene elementos de significativo valor para la integración de una política pública coherente con las necesidades de pertinencia, cobertura, calidad y vinculación que este nivel educativo tiene en México y el mund. (Rubio Oca, 2007). 


\section{DISCUSIÓN}

Sin duda es pertinente la propuesta de fortalecer el papel de la CONAEVA como órgano facultado para sistematizar los esfuerzos de evaluación y acreditación de la educación superior mexicana que hasta ahora se encuentran dispersos, como se pudo observar en el segundo apartado de este artículo la CONEAU de Argentina ha realizado un trabajo exitoso de coordinación del que se puede aprender para consolidar una experiencia pertinente para México. No obstante, es importante señalar que se requiere generar una Ley General de Educación Superior o Ley de Educación Superior para que se le otorgue el sustento jurídico adecuado a las acciones que podría cumplir la CONAEVA para garantizar que la educación superior que se ofrezca en México sea coherente con las necesidades de calidad, pertinencia, cobertura y vinculación. Este es el reto mayor, es indispensable transitar hacia un nuevo marco jurídico que, como en el caso de Argentina, le otorgue a la evaluación y acreditación de la calidad un nivel normativo de carácter federal con posibilidades de limitar la irresponsabilidad de autoridades e instituciones de educación superior.

Para concluir con esta reflexión, será de especial relevancia que esa Ley General de Educación Superior o Ley de Educación Superior (o como se le quiera llamar), deberá definir el propósito de la educación superior en México, en otras palabras ¿para qué educamos en las IES mexicanas? Como se puede deducir de esta pregunta, el centro de la propuesta implica la necesaria e ineludible exigencia de establecer el proyecto educativo de la nación. De otro modo, la evaluación y acreditación serán sólo mecanismos burocráticos y mercadológicos y no una función educativa sustancial e inescapable para la consolidación del proceso formativo de los mexicanos a fin de que sean profesionistas comprometidos con la transformación del país.

La necesidad de no limitarse a los aspectos puramente económicos del desarrollo, sino a considerar la realización personal del individuo como una de las objetivos esenciales de la educación y la formación.

\section{REFERENCIAS}

Aboites, H. (2012). La medida de una nación: los primeros años de la evaluación en México. México: CLACSO-ÍTACA

Aboites, H. (2003). "Actores y políticas en la educación superior mexicana: las contradicciones del pacto de modernización empresarial." En Mollis, Marcela (compiladora) (2003) Las Universidades en América Latina: ireformadas o alteradas? La cosmética del poder financiero. Buenos Aires: CLACSO-Agencia Sueca de Desarrollo Internacional.

Aréchiga, H. y Llarena de Thierry, R. (2003). "Antecedentes, situación actual y perspectivas de la evaluación y acreditación de la educación superior en México." En La evaluación y la acreditación en la educación superior en América Latina y el Caribe. Venezuela: IESALC-UNESCO. 
De la Garza, J. (2008). "Evaluación y acreditación de la educación superior en América Latina y el Caribe" en Tünnermann Bernheim, Carlos (editor) Federación de Instituciones Mexicanas Particulares de Educación Superior (2013) Página de Internet de la FIMPES. México: www.fimpes.org.mx consultada el 15 de octubre de 2013.

Fernández, N. (2003). "Evaluación y acreditación en la educación superior argentina" en La evaluación y la acreditación en la educación superior en América Latina y el Caribe. Venezuela: IESALC-UNESCO.

Fernández, V. (2009). "La necesidad de pactos de Estado en la política española", en la revista SEECI, no. 20, Madrid.

Gago, A. (2012). Evaluación, certificación y acreditación en la educación superior en México. Hacia la integración del subsistema para evaluar la educación superior. México: ANUIES.

Gago, A. (2002). Apuntes acerca de la evaluación educativa. México: SEP.

Korniejczuk, V. A. (2002). La acreditación de la educación superior presencial y a distancia en Estados Unidos y México. México: Universidad de Montemorelos.

López, R. (2012). "¿Es el Programa Integral de Fortalecimiento Institucional (PIFI) una moda?" en Revista de la Educación Superior (No. 163, Vol. 41, julio-septiembre de 2012) México: ANUIES

Peña, B. (2003). "Reflexiones acerca de la enseñanza en la sociedad actual", en la revista SEECI, no. 10, Madrid.

Rubio, J. (2007). "La evaluación y acreditación de la educación superior en México: un largo camino por recorrer." En revista Reencuentro, número 50, diciembre de 2007. México: UAM-Xochimilco.

\section{Braulio González Vidaña}

Licenciado en Ciencias Políticas y Administración Pública por la Facultad de Ciencias Políticas y Sociales de la UNAM, con mención honorifica, maestro en Ciencias Políticas maestro en Ciencias de la Educación, es candidato al Doctorado en Pensamiento Complejo. Vicerrector Académico en la Universidad del Claustro de Sor Juana. Ha colaborado con la Federación de Instituciones Mexicanas Particulares de Educación Superior, A.C. (FIMPES) desempeñando responsabilidades como visitador evaluador; miembro de la Comisión Permanente de Dictaminación de la FIMPES y Coordinador de la Comisión Permanente de Dictaminación de FIMPES. 